\title{
SCHÄDEL-HIRN-TRAUMATA
}

\section{Noch Jahre später Sterblichkeit erhöht}

Patienten, die ein Schädel-Hirn-Trauma (SHT) erlitten haben, haben noch Jahre später ein erhöhtes Sterberisiko, ergab eine schottische Studie mit 757 SHT-Patienten. Als Kontrollen dienten Personen, die aus anderen Gründen hospitalisiert waren bzw. Gesunde. Innerhalb von 13 Jahren verstarben $40 \%$ der Schädel-Hirn-Verletzten, $28 \%$ der aus anderen Gründen Hospitalisierten und 19\% der gesunden Vergleichsgruppe. Besonders kritisch ist das erste Jahr nach dem Ereignis. Hier gilt - wenig überra- schend -, dass Patienten mit leichtem Trauma eher überleben als solche mit schwereren Verletzungen. Doch nach dem ersten Jahr bleibt die Sterblichkeit bei Patienten mit durchgemachtem Schädel-Hirn-Trauma erhöht, unabhängig von der Schwere der ursprünglichen Verletzung. In der Altersgruppe von 15 bis 54 Jahren war die Sterblichkeit sechsmal höher als bei Personen ohne Schädelverletzungen.

J Neurol Neurosurg Psychiatry, 31 January 2011 DO 10.1136/jnnp.2010.222232

\section{ARZNEIMITTELALLERGIE}

\section{Tödliche Gefahr auf der Sonnenbank}

Manche Menschen legen sich auf eine Sonnenbank, weil sie sich die Linderung ihres Hautleidens versprechen. Doch kann diese Art der Selbstbehandlung lebensgefährlich werden, warnen Dermatologen der Indiana University School of Medicine: Eine Patientin hatte nach der Einnahme von Ibuprofen einen leichten Hautausschlag bekommen. Nach einer Selbstbehandlung im Sonnenstudio entwickelte sie eine toxische epidermale Nekrolyse (TEN), mit schwerer Blasenbildung und Blutdruckabfall. TEN führt in

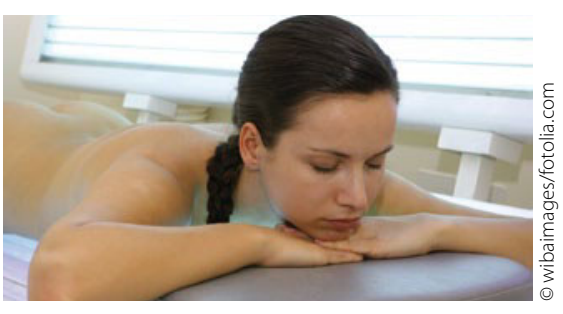

Gefahr für Haut und Leben?

20\% der Fälle zum Multiorganversagen und zum Tode.

Arch Dermatol. 2011;DOI: 10.1001/archdermatol.2011.13

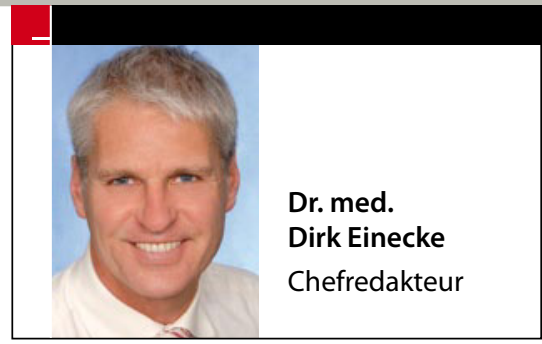

\section{LUNGENKREBS}

\section{Wenn Raucher nicht mehr qualmen wollen}

Wenn Raucher spontan ihr Nikotinlaster aufgeben, ist dies nicht unbedingt ein gutes Zeichen, besonders dann, wenn sie schon mehrere vergebliche RauchstoppVersuche hinter sich haben und sich plötzlich mit dem Rauchverzicht ganz leicht tun. Diese Patienten könnten einen - noch asymptomatischen Lungenkrebs haben. Im Philadelphia Veterans Affairs Medical Center wurden 115 (Ex-)Raucher mit Lungenkarzinom befragt. 48\% dieser Patienten gaben an, sie hätten das Rauchen vor der Diagnose aufgegeben. Nur $11 \%$ hatten zu diesem Zeitpunkt bereits Symptome, die auf den Lungenkrebs zurückzuführen waren. $31 \%$ berichteten, sie hätten keine Probleme mit dem Rauchverzicht gehabt. Zwischen der Aufgabe des Rauchens und der Diagnose Lungenkrebs lagen bei diesen Patienten durchschnittlich 2,7 Jahre. Journal of Thoracic Oncology, 2011; 6 (3): 517 DOI: 10.1097/JTO.0b013e318208c7da

\section{NACH DEM NOTFALLTRAINING ÜBERRASCHEND KOMPETENT \\ Knirpse als Ersthelfer}

Können Fünfjährige Erste Hilfe leisten? Schwedische Forscher wollten herausfinden, ob ein Notfalltraining schon im Kindergartenalter sinnvoll ist. Sie ließen zehn Vier- bis Fünfjährige an einem Erste-HilfeKurs teilnehmen und testeten zwei Monate nach Kursende deren Fähigkeiten in einem gestellten Unfallszenario. Die Mehrheit der Knirpse zeigte sich überraschend kompetent: $70 \%$ schätzten die Bewusstlosigkeit des fiktiven Unfallopfers richtig ein und hatten die Notrufnummer parat. $60 \%$ erkannten, ob das Opfer atmete, und $40 \%$ wussten, wie man Verunfallte korrekt lagert und deren Atemwege freimacht. Beginnt das Erste-Hilfe-Training bereits im Kindergarten und wird im Schulalter verstärkt, würden langfristig mehr Menschen ermutigt, im Ernstfall Erste Hilfe zu leisten.

Scandinavian Journal of Trauma, Resuscitation and Emergency Medicine 2011,19:13; doi:10.1186/17577241-19-13

\section{www.SpringerMedizin.de}

Unter die Lupe genommen: Infektiologie, Diabetes, Schmerz

Einmal abonniert, regelmäßig informiert! Bestellen Sie jetzt auf SpringerMedizin.de einen unserer drei neuen Newsletter und erfahren Sie alles Wichtige zu Diagnostik, Therapie und Fortbildung. http://registrierung.springermedizin.de/sprsso/home/ content-142701.html 